\section{Pourrait-on traiter le diabète de type 1 en stimulant les lymphocytes $\mathbf{T}$ régulateurs?}

Yenkel Grinberg-Bleyer, Audrey Baeyens, Eliane Piaggio, Benoit L. Salomon
13, immunology, immunopathology, immunotherapy, CNRS/UPMC UMR7211, Inserm U959,

Hôpital Pitié-Salpêtrière, Paris, 75013 France.

elianepiaggio@yahoo.com

benoit.salomon@upmc.fr

દ. Piaggio et B.L.Salomon : participation égale.
Les lymphocytes $\mathrm{T}$ régulateurs, cellules essentielles à la régulation de l'autoimmunité d'une attaque du système immunitaire contre son propre hôte, conséquence d'une rupture de la tolérance au soi. Elles sont souvent associées à des signes cliniques graves altérant la qualité de vie des patients. La prévalence de ces pathologies atteint 5 à $10 \%$ de la population dans les pays occidentaux. Les maladies auto-immunes constituent donc un problème de santé publique majeur. Malheureusement, la plupart de ces pathologies ne peuvent être guéries; les traitements disponibles ne sont souvent que palliatifs et doivent être administrés à vie. L'étude de la physiopathologie des maladies auto-immunes et la découverte de nouveaux traitements présentent donc un intérêt indéniable. Les lymphocytes $T$ régulateurs $C D 4^{+} \mathrm{CD} 25^{+}$ FoxP3 $^{+}\left(T_{\text {reg }}\right)$, qui représentent 5 à $15 \%$
Les maladies auto-immunes résultent des lymphocytes T CD4 $4^{+}$dans les organes lymphoïdes, sont des acteurs majeurs de la régulation des réponses immunes [ 1 , 2]. Ils participent au maintien de la tolérance au soi, prévenant ainsi les maladies auto-immunes. En effet, un déficit sévère en $T_{\text {reg }}$ dû à la présence de mutations dans Foxp3, gène codant pour un facteur de transcription essentiel au développement et à la fonction des $T_{\text {reg, }}$ conduit à un syndrome auto-immun létal chez l'homme et la souris [3]. De plus, les souris non-obese diabetic (NOD) - modèles de diabète de type 1 - développent, lorsqu'elles sont déficientes en $T_{\text {reg }}$, un diabète accéléré et aggravé [4]. À l'inverse, l'injection de $T_{\text {reg }}$ spécifiques d'antigènes pancréatiques permet de prévenir le diabète et même d'induire une rémission des signes cliniques [5]. Le rôle protecteur des $\mathrm{T}_{\text {reg }}$ a été observé dans la plupart des pathologies auto-immunes étudiées. C'est pourquoi, depuis plusieurs années, de nombreuses équipes s'intéressent à l'utilisation des $\mathrm{T}_{\text {reg }}$ en biothérapies. À ce jour, nous ne savons pas produire de $T_{\text {reg spécifiques d'auto- }}$ antigènes d'un tissu cible en conditions de grade clinique, ce qui limite l'intérêt d'une thérapie cellulaire. Une alternative thérapeutique consisterait à administrer des molécules capables de stimuler préférentiellement le compartiment des $T_{\text {reg }}$ du patient pour augmenter leur capacité à contrôler une autoréactivité pathogène. Récemment, nous avons montré que l'interleukine-2 (IL-2) et le tumor necrosis factor (TNF) sont capables de stimuler les $T_{\text {reg }}$ spécifiquement dans le pancréas enflammé des souris NOD, jouant ainsi un rôle régulateur dans le diabète.

\section{Propriétés régulatrices de l'IL-2 dans le diabète par ses effets sur les Treg} L'IL-2 est l'un des facteurs essentiels à la survie et la fonction des $T_{\text {reg. }}$. Ainsi, les souris déficientes en IL-2 présentent un fort déficit en $T_{\text {reg }}$ et meurent d'un syndrome auto-immun généralisé [6]. En 2008, nous avons mis en évidence un déficit local en

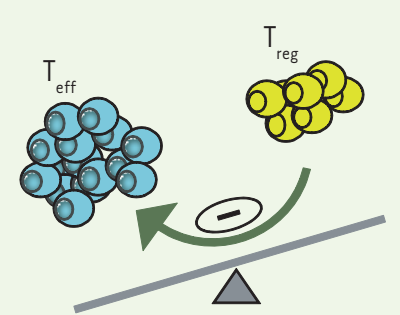

Auto-immunité

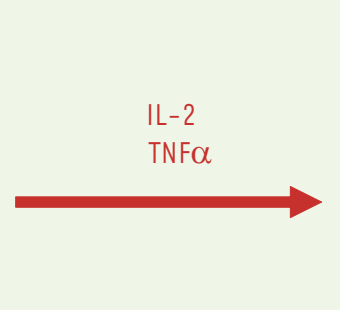

IL-2

TNFO

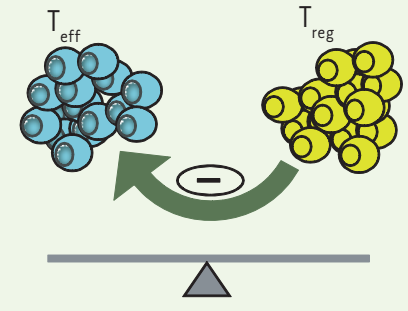

Tolérance
Figure 1. La stimulation des $T_{\text {reg }}$ par l'IL-2 ou le TNF $\alpha$ rétablit l'équilibre inflammation/tolérance dans le diabète. Lors du développement du diabète de type 1 , certains lymphocytes $T$ effecteurs $\left(T_{\text {eff }}\right.$ ) autoréactifs s'activent fortement, entraînant une inflammation destructrice dans le pancréas. L'IL-2 et le TNF $\alpha$ pourraient favoriser un rétablissement de l'équilibre entre $T_{\text {eff }}$ et $T_{\text {reg }}$ et un retour à la tolérance en stimulant préférentiellement les $T_{\text {reg }}$ infiltrant le pancréas. IL-2 dans le pancréas des souris NOD, entraînant une survie diminuée des $T_{\text {reg }}$ dans ce tissu, ce qui pourrait précipiter la destruction des cellules $\beta$ productrices d'insuline [7]. De façon intéressante, l'injection prolongée de faibles doses d'IL-2 à des souris NOD jeunes entraîne une réduction importante de l'incidence du diabète, associée à une augmentation du nombre et de la survie 
des $T_{\text {reg }}$ infiltrant le pancréas. Nous avons ensuite testé un éventuel effet curatif de I'IL-2 chez des souris NOD nouvellement diabétiques. De façon remarquable, un traitement de seulement 5 jours avec des faibles doses d'IL-2 induit une rémission de l'hyperglycémie chez près de $60 \%$ des souris [8]. Chez la majorité de ces animaux, la glycémie est normalisée à long terme (plus de 10 semaines après le traitement). Toutefois, il est notable que ce traitement ne fonctionne pas si la glycémie des souris est supérieure à $350 \mathrm{mg} / \mathrm{dL}$, mettant en évidence la nécessité, pour espérer avoir une efficacité thérapeutique, d'agir à des temps précoces après le diagnostic, quand la quantité résiduelle de cellules $\beta$ productrices d'insuline est suffisante pour réguler la glycémie. Nous avons ensuite étudié l'implication des $\mathrm{T}_{\text {reg }}$ dans ce traitement. D'une part, nous avons observé que l'administration de ces faibles doses d'IL-2 induit une augmentation significative de la proportion de $T_{\text {reg }}$ dans le pancréas. Celle-ci est associée à une réduction de l'agressivité des lymphocytes $T$ effecteurs ( $\left.T_{\text {eff }}\right)$ diabétogènes qui sécrètent moins d'interféron- $\gamma$ (IFN- $\gamma$ ), cytokine toxique pour les cellules $\beta$ productrices d'insuline. D'autre part, cette thérapie par l'IL-2 s'avère inefficace chez des souris NOD génétiquement déficientes en $T_{\text {reg. }}$. Ceci montre que l'IL-2 permet une rémission du diabète clinique par un effet local sur les lymphocytes infiltrant le pancréas. II est important de remarquer que ces faibles doses thérapeutiques d'IL-2 ne semblent pas avoir d'effet systémique. En effet, dans les tissus lymphoïdes, le traitement ne modifie pas la proportion de $T_{\text {reg }}$ et n'a que peu d'effet sur la signature ARN des $T_{\text {reg }}$ et $T_{\text {eff }}$ déterminée par analyse des transcriptomes. Ces résultats et les données chez l'homme suggèrent une relative innocuité de l'administration de faibles doses d'IL-2. Jusqu'à ce jour, l'IL-2 a surtout été utilisée à fortes doses dans certains cancers et maladies infectieuses pour stimuler le système immunitaire, avec des effets thérapeutiques modérés voire discutables. Dans le futur, cette même cytokine pourrait être utilisée à faible dose pour traiter certaines maladies auto-immunes.
Un mécanisme de rétrocontrôle de l'auto-immunité impliquant le TNF $\alpha$ Les $\mathrm{T}_{\text {eff }}$ étant les principales cellules productrices d'IL-2, ils pourraient être capables de stimuler les $T_{\text {reg. Nous avons }}$ étudié cette question dans un modèle murin de diabète de type 1 par des expériences de transfert adoptif de $T_{\text {reg }}$ et $T_{\text {eff }}$ spécifiques d'antigènes pancréatiques. De façon remarquable, le cotransfert de $T_{\text {eff }}$ diabétogènes entraîne une très forte augmentation de l'expansion des $T_{\text {reg }}$ dans les ganglions pancréatiques et le pancréas, d'un facteur 10 à 15 par rapport aux souris qui n'ont reçu que des $\mathrm{T}_{\text {reg }}$ [9]. Nous avons ensuite étudié les implications physiopathologiques de ce phénomène dans le diabète. Lorsque les $T_{\text {reg }}$ sont transférés seuls, ils ne permettent pas de prévenir l'apparition du diabète induit par l'injection de $T_{\text {eff }}$ diabétogènes 3 semaines plus tard. En revanche, si les $T_{\text {reg }}$ sont initialement cotransférés avec les $T_{\text {eff, }}$, les souris sont protégées à long terme contre l'induction de la maladie. Ainsi, des $T_{\text {eff }}$ diabétogènes limitent leur propre effet pathogène en activant les

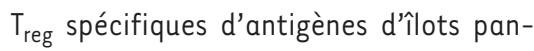
créatiques. De façon inattendue, cet effet stimulant des $T_{\text {eff }}$ sur les $T_{\text {reg }}$ est indépendant de I'IL-2, et partiellement dépendant du TNFo. En effet, l'augmentation de l'expansion des $T_{\text {reg }}$ par les $T_{\text {eff }}$ est significativement réduite par des traitements qui neutralisent cette cytokine. Ces résultats pourraient permettre de révéler le mécanisme responsable de la composante immuno-régulatrice du TNF $\alpha$. En effet, cette cytokine est surtout connue pour ses propriétés proinflammatoires et les effets bénéfiques des anti-TNF dans le traitement de certaines maladies auto-immunes comme la polyarthrite rhumatoïde. Cependant, des données chez l'homme et la souris montrent au contraire un rôle protecteur du TNF $\alpha$ dans d'autres maladies auto-immunes, comme la sclérose en plaques $[10,11]$. Nos données suggèrent que cette facette immuno-régulatrice du TNF $\alpha$ pourrait s'expliquer par la capacité de cette cytokine à stimuler les $\mathrm{T}_{\text {reg. }}$.
L'identification de facteurs capables de stimuler de façon préférentielle le compartiment des $T_{\text {reg }}$ dans les tissus cibles des maladies auto-immunes peut présenter un intérêt thérapeutique majeur. Nos données suggèrent que I'IL-2 et le TNF $\alpha$ ont un rôle protecteur dans le diabète de type 1 par leur capacité à favoriser l'expansion des $T_{\text {reg }}$ dans le pancréas. La rémission du diabète obtenue après administration d'IL-2 chez la souris NOD nous conduit à proposer un essai clinique à visée thérapeutique chez des patients atteints de diabète de type 1 récemment diagnostiqués. $\diamond$

Could we cure type 1 diabetes

by stimulating $\mathrm{T}_{\text {reg }}$ ?

\section{CONFLIT D'INTÉRÊTS}

Les auteurs déclarent n'avoir aucun conflit d'intérêts concernant les données publiées dans cet article.

\section{RÉFÉRENCES}

1. Tang Q, Bluestone JA. The Foxp3+ regulatory T cell: a jack of all trades, master of regulation. Nat Immunol $2008 ; 9: 239-44$.

2. Salomon BL. Les lymphocytes T régulateurs $\mathrm{CD}^{+} \mathrm{CD}_{25} 5^{+}$: vers une immuno-modulation thérapeutique? Med Sci (Paris) $2002 ; 18: 1066-8$.

3. Wildin RS, Ramsdell F, Peake J, et al. X-linked neonatal diabetes mellitus, enteropathy and endocrinopathy syndrome is the human equivalent of mouse scurfy. Nat Genet $2001 ; 27$ : 18-20.

4. Salomon B, Lenschow DJ, Rhree L, et al. B7/CD28 costimulation is essential for the homeostasis of the $\mathrm{CD} 4^{+} \mathrm{CD} 25^{+}$immunoregulatory $\mathrm{T}$ cells that control autoimmune diabetes. Immunity $2000 ; 12: 431-40$.

5. Tang $Q$, Henriksen KJ, Bi M, et al. In vitro-expanded antigen-specific regulatory $T$ cells suppress autoimmune diabetes. J Exp Med 2004 ; 199 : 1455-65

6. Sadlack B, Merz H, Schorle H, et al. Ulcerative colitislike disease in mice with a disrupted interleukin-2 gene. Cell $1993 ; 75: 253-61$.

7. Tang Q, Adams Jy, Penaranda C, et al. Central role of defective interleukin-2 production in the triggering of islet autoimmune destruction. Immunity 2008; 28 : 687-97.

8. Grinberg-Bleyer Y, Baeyens A, You S, et al. IL-2 reverses established type 1 diabetes in NOD mice by a local effect on pancreatic regulatory T cells. J Exp Med $2010 ; 207: 1871-8$.

9. Grinberg-Bleyer $\mathrm{Y}$, Saadoun D, Baeyens A, et al. Pathogenic T cells have a paradoxical protective effect in murine autoimmune diabetes by boosting $\mathrm{T}_{\text {regs. }}$. Clin Invest $2010 ; 120: 4558-68$.

10. TNF neutralization in MS : results of a randomized, placebo-controlled multicenter study. The Lenercept multiple sclerosis study group and the University of British Columbia MS/MRI analysis group. Neurology $1999 ; 53: 457-65$.

11. Liu J, Marino MW, Wong $G$, et al. TNF is a potent antiinflammatory cytokine in autoimmune-mediated demyelination. Nat Med $1998 ; 4: 78-83$. 